\title{
Aprendizaje de la circunferencia aplicando el Modelo Van Hiele en estudiantes de undécimo grado de Educación Secundaria de Río San Juan
}

\author{
Misael Ariel Suárez Martínez' \\ Joel Antonio Martínez Ortiz² \\ Eugenio Casimiro López Mairena
}

\section{Resumen}

C sta investigación ha analizado el aprendizaje de la circunferencia aplicando el modelo de Van Hiele en E estudiantes de undécimo grado. Fue un estudio cualitativo con enfoque fenomenológico con la participación de 12 estudiantes de undécimo grado, durante el segundo semestre y un profesor de matemática del Instituto "Miguel Larreynaga", El Castillo, Río San Juan. El análisis se sustentó en la observación en el aula, test actitudinal y encuesta. Los resultados indican que el modelo de Van Hiele es un enfoque idóneo para la enseñanza de la circunferencia que puede adaptarse a cualquier entorno de aprendizaje, ayudando de manera efectiva a la disminución de las dificultades en el aprendizaje y dotando al estudiantado de herramientas indispensables para el aprendizaje por descubrimiento, la resolución de problemas y actitud ante los desafíos en los diferentes niveles del modelo.

Palabras clave: Modelo de Van Hiele; Circunferencia; Aprendizaje por descubrimiento.

\section{Abstract}

This research has analyzed the learning of the circumference applying Van Hiele Model in eleventh grade students. It was a qualitative study with a phenomenological approach with the participation of 12 students from eleventh grade during the second semester and a teacher of mathematics at "Miguel Larreynaga" Institute, El Castillo, Río San Juan. The analysis was based on the observation in the classroom, attitudinal test and survey. The results indicate that the Van Hiele model is an ideal approach to the teaching of circumference that can be adapted to any learning environment, helping effectively to reduce learning difficulties and providing the students with indispensable tools for learning by discovery, problem solving and attitude to the challenges at the different levels of the model.

Keywords: Van Hiele model; Circumference; Learning by discovery.

\footnotetext{
1 Licenciado en Ciencias de la Educación con mención en Matemática. Profesor de Matemáticas del Ministerio de Educación, El Castillo, Río San Juan. correo: suarezalways@gmail.com

2 Licenciado en Ciencias de la Educación con mención en Matemática. . Profesor de Matemáticas del Ministerio de Educación, El Castillo, Río San Juan correo: jomartinezamo@gmail.com

3 Doctor en Innovación en la formación. Vicerrector de la Universidad de las Regiones Autónomas de la Costa Caribe Nicaragüense-Recinto Universitario Nueva Guiea.Correo: eugenio.lópez@uraccan.edu.ni
}

Recibido:03/05/2017 - Aprobado: 12/06/2017 


\section{Introducción}

La enseñanza de la circunferencia, corresponde a la unidad de geometría analítica del programa de matemática de undécimo grado Educación Secundaria de Nicaragua. El aprendizaje de esta temática exige conocimientos previos de geometría básica (Euclidiana) y álgebra. Durante los años anteriores no ha sido posible alcanzar aprendizaje satisfactorio en el estudiantado por la implementación de modelos y enfoques que no contribuyen al aprendizaje por descubrimiento, por ser un tema de la última unidad del programa de matemática, no se completa la carga horaria establecida por el Ministerio de Educación, lo que conlleva a abordar nociones y en algunos casos no se desarrolla la temática.

Haciendo propio el compromiso de contribuir en el proceso y evolución del aprendizaje de la matemática, esta investigación pretende dotar al profesorado de herramientas que le permitan interrelacionar los diversos saberes para que el ambiente de estudio sea muy dinámico y participativo a través de la resolución de problemas contextualizados, tomando como herramienta de apoyo distintos tipos de software matemáticos que fortalezcan y despierten el interés del estudiantado en el aprendizaje de la circunferencia.

\section{Revisión literaria}

La matemática como disciplina básica del programa de estudio de Educación Secundaria, está en caminada a prever de conocimientos que conduzcan a formular y resolver diversos problemas del entorno. Las capacidades y niveles de aprendizaje son diferenciados, pero debe tratarse de manera individualizada de manera que permitan alcanzar los objetivos propuestos, por tal razón se propone el Modelo de Van Hiele (Vargas y Araya, 2013). El modelo de Van Hiele ayuda a explicar cómo, en el proceso de aprendizaje de la geometría, el razonamiento geométrico de los estudiantes transcurre por una serie de niveles.

En este sentido, el aprendizaje será posible cuando el profesorado y el estudiantado logren armonizar y establecer un ambiente de desafíos, en el cual, cada uno tenga el espacio de presentar sus saberes, inquietudes, formas de percibir el aprendizaje per se y por ende, facilitar el espacio para la nueva construcción del mismo. Por ello, resulta oportuna la afirmación de Fouz y De Donosti
(2005), el punto clave en la utilización del modelo de Van Hiele es precisamente la evaluación. En el marco de este modelo interesa la valoración de un individuo tomando en cuenta las razones por las que dio determinada respuesta. A partir de esto, los mismos autores indican que lo más recomendable para la evaluación, a la luz de este modelo, es la combinación de la entrevista y el test.

Tomando en cuenta que la geometría está presente en la mayoría de situaciones que se presentan en nuestro entorno, por tal razón, debemos de valernos de cada elemento que permita facilitar un aprendizaje de la manera más sencilla y aplicada a la realidad, es indispensable el seguimiento permanente del proceso de enseñanza-aprendizaje desde la parte motivacional del estudiantado hasta la parte pedagógica, deben estar contenidas en la planificación de las sesiones de clase en la cual permita mantener involucrado a los estudiantes desde inicio del curso hasta su final, para que el modelo tenga la efectividad con la cual se especifica en cada uno de los niveles. Por consiguiente, Fouz y De Donosti (2005) en su análisis señalan las características fundamentales que se desarrollan en el modelo de Van Hiele desde la perspectiva del aprendizaje, véase tabla 1.

Tabla 1: Niveles de razonamiento del modelo de Van Hiele

\begin{tabular}{|c|l|}
\hline Niveles & \multicolumn{1}{|c|}{ Características } \\
\hline 0 & $\begin{array}{l}\text { Contextualizan los conocimientos, llevándolos a una revisión } \\
\text { de aprendizajes empíricos o científicos que haya construido } \\
\text { con anterioridad. }\end{array}$ \\
\hline 1 & $\begin{array}{l}\text { Describe figuras por sus propiedades pero no de relacionar } \\
\text { unas propiedades con otras o unas figuras con otras. }\end{array}$ \\
\hline 2 & $\begin{array}{l}\text { Clasificaciones lógicas de manera formal, reconoce cómo unas } \\
\text { propiedades derivan de otras, estableciendo relaciones entre } \\
\text { propiedades y las consecuencias de esas relaciones. }\end{array}$ \\
\hline 3 & $\begin{array}{l}\text { Llega a los mismos resultados mediante proposiciones o } \\
\text { premisas distintas, realiza demostraciones para obtener un } \\
\text { mismo resultado. }\end{array}$ \\
\hline 4 & $\begin{array}{l}\text { Se puede trabajar la Geometría de manera abstracta sin nece- } \\
\text { sidad de ejemplos concretos, alcanzándose el más alto nivel } \\
\text { de rigor matemático. }\end{array}$ \\
\hline
\end{tabular}

\section{Materiales y métodos}

Esta investigación es de tipo cualitativa y su diseño se define como fenomenológico, porque, trata de analizar, describir y observar las dificultades de compresión en el aprendizaje de la circunferencia, permitiendo establecer una propuesta didáctica 
que satisfaga las necesidades de aprendizaje del estudiantado. El estudio se realizó durante el segundo semestre del año 2016, su verdadero potencial radica en la capacidad para generar hipótesis y descubrimientos, en centrar su interés en un individuo o situación, en su flexibilidad y aplicabilidad a situaciones naturales (Latorre et al., 1996, p. 26).

Participaron 12 estudiantes de undécimo grado, siete hombres que corresponden al 58\% y cinco mujeres que representan el $42 \%$, incluyendo un profesor que imparte la disciplina de matemática en el Instituto Miguel Larreynaga, de la comunidad Boca de Sábalos. Para la recopilación de la información se suministró un Test Cognitivo y Encuesta al estudiantado, observación directa en el aula de clase y entrevista dirigida al profesor. En el caso del análisis de la entrevista y observación se llevó a efecto mediante criterios previamente definidos que incluye datos cualitativos y cuantitativos, permitiendo de manera efectiva la elaboración de una propuesta didáctica para el aprendizaje de la circunferencia a través del Modelo de Van Hiele con el propósito de contribuir a satisfacer las necesidades de aprendizaje desde el enfoque de razonamiento matemático.

El procesamiento de la información se realizó con el software Statistical Package for the Social Sciences (SPSS), que ayudó a realizar un análisis descriptivo del test y la encuesta, y de forma manual las observaciones, también se usó el Microsoft Excel para presentar la información a través de gráficas y así aprovechar toda la información relevante, veraz y objetiva, que permitió que los resultados no tengan sesgo alguno.

\section{Resultados y discusión}

El aprendizaje de la ecuación de la circunferencia potencia grandes resultados en el estudiantado siguiendo de manera significativa el modelo de Van Hiele, fortalece las capacidades del docente, puesto que la implementación de cada nivel exige de tener dominio de cada tópico abordado en la ecuación de la circunferencia. Queda en manifiesto que la apropiación del modelo y su implementación permite obtener resultados significativos en el estudiantado ya que son capaces de proponer problemas y al mismo tiempo resolverlos mediante diversos métodos que le ayudan a comprobar los resultados.

En el proceso de la investigación se aplicó una prueba antes de validar la unidad didáctica y después de haber hecho la intervención, los resultados fueron satisfactorios, los que presentamos en la tabla 2.

Tabla 2: Resultados de la prueba antes y después de la intervención pedagógica

\begin{tabular}{|c|c|c|c|c|}
\hline \multirow{2}{*}{ ÍTEMS } & \multicolumn{2}{|c|}{ Prueba inicial } & \multicolumn{2}{|c|}{ Prueba final } \\
\hline & Acierto & Errores & Acierto & Errores \\
\hline 1. Simplificando la expresión $12-6 \div 3+5 \times 2=$. & 2 & 10 & 9 & 3 \\
\hline $\begin{array}{l}\text { 2. una circunferencia tiene centro } C(5,-3) \text { es tangente a la recta } 12 x-5 y-36=0 \text {, } \\
\text { mediante la fórmula }\end{array}$ & 1 & 11 & 8 & 4 \\
\hline $\begin{array}{l}\text { 3. Calcular el área de la región sombreada. } A B C D \text { es un cuadrado cuyo lado } \\
\text { mide } 6 \mathrm{~cm} \text {. }\end{array}$ & 0 & 12 & 8 & 4 \\
\hline $\begin{array}{l}\text { 4. Dados los siguientes pares ordenados A }(3,2) ; B(-2,0) \text {; C (-4,4), representa } \\
\text { los puntos en el sistema cartesiano, al unir los puntos por segmentos, que fi- } \\
\text { gura resulta. }\end{array}$ & 7 & 5 & 11 & 1 \\
\hline $\begin{array}{l}\text { 5. Sume los términos de cada columna y escriba los resultados en cada espacio } \\
\text { del final del cuadro. }\end{array}$ & 0 & 12 & 12 & 0 \\
\hline
\end{tabular}




\begin{tabular}{|c|c|c|c|c|}
\hline \multirow{2}{*}{ ÍTEMS } & \multicolumn{2}{|c|}{ Pruebas inicial } & \multicolumn{2}{|c|}{ Pruebas final } \\
\hline & Acierto & Errores & Acierto & Errores \\
\hline 6. La tercera parte de "t" menos 4 es igual 12. & 3 & 9 & 7 & 5 \\
\hline $\begin{array}{l}\text { 7. Los elementos de la circunferencia son: (cuerda, radio, tangente, secante, } \\
\text { diámetro), escribe en la figura el nombre de cada elemento. }\end{array}$ & 3 & 9 & 10 & 2 \\
\hline $\begin{array}{l}\text { 8. Una vaca está atada a un árbol que se encuentra en un prado. Si la cuerda } \\
\text { tiene } 12 \mathrm{~m} \text { de longitud, ¿cuál es el área de prado que se halla al alcance del } \\
\text { animal? }\end{array}$ & 8 & 4 & 11 & 1 \\
\hline 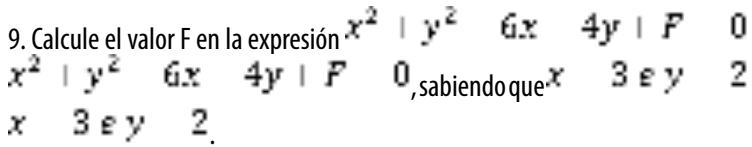 & 1 & 11 & 7 & 5 \\
\hline $\begin{array}{l}\text { 10. El diámetro de una circunferencia tiene las coordenadas } A(2,-1) \text { y } B(5,3) \text {. } \\
\text { Calcular la longitud del radio. }\end{array}$ & 0 & 12 & 9 & 3 \\
\hline Porcentajes Totales & $20.84 \%$ & $79.16 \%$ & $76.7 \%$ & $23.3 \%$ \\
\hline
\end{tabular}

El estudiantado se integra activamente en el proceso de aprendizaje, motivándose entre ellos, la confianza y estrecha relación docente-estudiante contribuye a que las clases se desarrollen en un entorno agradable. Las actividades desarrolladas en la intervención pedagógica desarrollan el pensamiento matemático, partiendo de los conocimientos previos y situaciones cotidianas.

El aprendizaje de la circunferencia se complementó con el uso de recursos didácticos y tecnológicos, utilizando software matemático que permitió al estudiantado corroborar las respuestas de las actividades propuestas. Otro aspecto fundamental a retomar es la evaluación pertinente de cada sesión de clase durante la intervención a través aplicación de la propuesta didáctica, la realización de actividades colectivas, exposiciones, paneles y ejercicios en la pizarra contribuyeron en el estudiantado a forjar disciplina y dedicación, al presentar de manera coherente sus resultados. Esto permitió la realización de evaluaciones diagnósticas y la permanencia en diversas formas de presentar el avance del aprendizaje, esto dio pautas para retomar las dificultades de aprendizaje y fortalecerlas en los reforzamientos escolares permanentes.

Dentro de las dificultades más sentidas por el estudiantado está el álgebra y ecuaciones de geometría básica, dado que debemos partir de las igualdades y fórmulas definidas para el planteamiento de ejercicios en la circunferencia, ya sean con centro o fuera del origen. Por tal razón la propuesta didáctica evidenció tres fases que le ayudan al estudiantado a entrelazar los conocimientos previos con los nuevos y así tener el conocimiento necesario para afrontar cualquier tipo de problema del tópico en estudio.

\section{Conclusiones}

En este artículo describimos lo relacionado con el proceso investigativo en el aprendizaje de la circunferencia aplicando el modelo de Van Hiele. Por tanto, se presentan las conclusiones obtenidas a continuación: El estudiantado posee poco dominio de conocimientos científicos-matemáticos relacionados con eliminación de signos, valor numérico, reducción de términos semejantes, lenguaje algebraico, así como hacer despejes, resolver potenciaciones y calcular radios o valores desconocidos a partir de datos y ecuaciones dadas, basado en los resultados del diagnóstico aplicado. 
De acuerdo con los resultados obtenidos en la intervención de la Unidad Didáctica con el modelo de Van Hiele aplicado en el aprendizaje de la Circunferencia, concluimos:

- Propició aprendizajes significativos al desarrollar sus fases y niveles teniendo en cuenta los ritmos y diversidad de aprendizajes de cada uno de ellos ya que el $76.7 \%$ logró asimilar significativamente los contenidos desarrollados.

- Precisó la planificación de actividades pertinentes para dar salida a los indicadores de logros y contenidos curriculares, lo que permitió que el estudiantado interactuara y se motivara al establecer las relaciones que existían entre lo aprendido y lo que tenía en su ambiente, además de encontrar sentido en el usos de estos conocimientos en la vida cotidiana.

- El uso de herramienta digital como "GeoGebra" permitió dinamizar, afianzar y aclarar dudas surgidas en el proceso enseñanza aprendizaje en el estudiantado, ya que a través de ella pudieron trazar diferentes gráficas: Circunferencias, rectas, entre otras.

\section{Bibliografía}

Albert, M. E., \& Ros, M. Z. (2008). Estrategias de aprendizaje y eLearning. Un apunte para la fundamentación del diseño educativo en los entornos virtuales de aprendizaje.: Consideraciones para la reflexión y el debate. Introducción al estudio de las estrategias y estilos de aprendizaje. RED: Revista de Educación a Distancia, (19), 3.

Álvarez, Á. R. S., Bello, S. L. G., Garrido, Y. C. P., \& Martínez, M. T. C. (2015). Aproximación al contenido de la preparación profesional para la dirección del proceso de enseñanza aprendizaje. Revista Didasc@lia: Didáctica y Educación. ISSN 2224-2643, 6(1), 205-218.

Arànega, S. (2013). De la detección de las necesidades de formación pedagógica a la elaboración de un plan de formación en la universidad.

Arceo, F. D. B., Rojas, G. H., \& González, E. L. G. (2002). Estrategias docentes para un aprendizaje significativo: una interpretación constructivista. McGraw-Hill.
Bran de Cacacho, J. (Dir.). (2009). Guía de adecuaciones curriculares. Guatemala. MINED. https:// www.mineduc.gob.gt/

Cabello Pardos, A., B., (2013). La Modelización De Van Hiele en el Aprendizaje Constructivo De La Geometría en Primero de la Educación Secundaria Obligatoria A Partir De Cabri. (Tesis Inédita de Doctorado) Universidad de Salamanca, Salamanca, España.

Carrasco, A., C., Nieto, L. J. B., \& Barona, E. G. (2015). El dominio afectivo en futuros Maestros de matemáticas en la Universidad de Extremadura. Paradigma, 29(2), 157-171.

Fouz, F., \& De Donosti, B. E. R. R. I. T. Z. E. G. U. N. E. (2005). Modelo de Van Hiele para la didáctica de la Geometría. Módulo 2: Teoría y Práctica en Geometría Objetivo N 3 Modelo de Van Hiele para la didáctica de la Geometría 91, 92. Obtenido de: http://cimm.ucr.ac.cr/

Gaitan, M., Lacayo, M., \& Flores, W., O. (2014). Comprensión del Aprendizaje de la parábola en undécimo grado aplicando el modelo de Van Hiele. Ciencia e Interculturalidad, 15(2), 21-33

Gamino-Carranza, A., \& Acosta-González, M. G. (2016). Curricular Model of México National Technological (Tecnológico Nacional de México). Revista Electrónica Educare, 20(1), 1-25.

García Retana, J Á; (2011). MODELO EDUCATIVO BASADO EN COMPETENCIAS: IMPORTANCIA Y NECESIDAD. Revista Electrónica "Actualidades Investigativas en Educación”, 11() 1-24. Recuperado de http:// www.redalyc.org/articulo.oa?id=44722178014

Godino, J. D. (2004). Didáctica de las Matemáticas Para Maestros. Granada: Universidad de Granada.

González, M. L. G., \& Arroyo, J. C. (2015). Implicación académica en matemáticas: percepcion de metas docentes y processos autorregularorios en estudiantes de bachillerato. Revista Ibero-Americana de Estudos em Educação, 9(4).

Latorre, A., del Rincón Igea, D., \& Arnal, J. (1996). Bases metodológicas de la investigación educativa. 
López, H. A. (2011). Programa de Estudio de Educación Secundaria Matemática 10mo y $11 \mathrm{mo}$. Managua : MINED.

Maradiaga M., \& Collado L., \& Valle M., \& Oporta J. C., \& Ojeda S. (Comps.). (2009). Transformación curricular, paradigmas y enfoques pedagógicos. Nicaragua. MINED.

Ortega, T., \& Pecharromán, C. (2015). Aprendizaje de conceptos geométricos através de visualizaciones. Avances de Investigación en Educación Matemática, 1(7).

Rechimont, E., Ferreyra, N., Andrada, N., \& Parodi, C. (2008). La comprensión de un concepto matemático y los registros de representación semiótica.

Reyes Castillo, W. V., \& Rosales Pereira, C. (2006). Propuesta Metodológica para la enseñanza y el aprendizaje de la firgura geométrica "las cönica". Nueva Guinea.

Rodríguez, S. V., Gutiérrez, C. O., \& Varela, O. A. (2015). Desarrollo y evaluación de un material didáctico multimedia para facilitar el apren- dizaje de matemáticas. Revista Facultad de Ciencias Básicas, 11(1).

Salvador, R. C. (1994). Diseño y evaluación de una propuesta curricular de aprendizaje de la geometría en enseñanza secundaria basada en el modelo de razonamiento de Van Hiele (Vol. 95). Ministerio de Educación. Obtenido de: http:// www.uv.es/

Sarasua, J., \&Arrieta, M. Propuesta De Categorización de los Objetivos Geométricos. Reanálisis De Los Niveles De Van Hiele Referidos A Las Figuras Planas. Obtenido de: https://scholar.google. com/

Vargas, G. V., \& Araya, R. G. (2013). El modelo de Van Hiele y la enseñanza de la geometría. Uniciencia, 27(1), 74-94. Obtenido de: http:// www.revistas.una.ac.cr/

Zapata-Ros, M. (2015). Teorías y modelos sobre el aprendizaje en entornos conectados y ubicuos. Bases para un nuevo modelo teórico a partir de una visión crítica del "conectivismo". Education in the Knowledge Society (EKS),16(1), 69-102. 